\title{
On localization in holomorphic equivariant cohomology
}

\author{
U. BRuZzo \\ Scuola Internazionale Superiore di Studi Avanzati, \\ Via Bonomea 265, 34136 Trieste, Italy; \\ Istituto Nazionale di Fisica Nucleare, Sezione di Trieste \\ E-Mail: bruzzo@sissa.it \\ V. Rubtsov \\ Université d'Angers, Département de Mathématiques, \\ UFR Sciences, LAREMA, UMR 6093 du CNRS, \\ 2 bd. Lavoisier, 49045 Angers Cedex 01, France; \\ ITEP Theoretical Division, 25 Bol. Tcheremushkinskaya, \\ 117259, Moscow, Russia \\ E-mail: Volodya.Roubtsov@univ-angers.fr
}

\begin{abstract}
We study a holomorphic equivariant cohomology built out of the Atiyah algebroid of an equivariant holomorphic vector bundle and prove a related localization formula. This encompasses various residue formulas in complex geometry, in particular we shall show that it contains as special cases Carrell-Liebermann's and Feng-Ma's residue formulas, and Baum-Bott's formula for the zeroes of a meromorphic vector field.
\end{abstract}

Date: November 2, 2018

2000 Mathematics Subject Classification: 32L10, 53C12, 53C15, 53D17, 55N25, 55N91

The authors gratefully acknowledge financial support and hospitality during the respective visits to Université d'Angers and SISSA. Support for this work was provided by MISGAM, by the I.N.F.N. project PI14 "Nonperturbative dynamics of gauge theories", the EINSTEIN Italo-Russian project "Integrability in topological string and field theory," the MATPYL Angers-SISSA project "Lie algebroids, equivariant cohomology, and topological quantum field and string theories," and the GEANPYL programme. 


\section{INTRODUCTION}

In his fundamental paper [5], Bott wrote formulas allowing to express the Chern numbers of a compact manifold $X$ in terms of the zeroes of a vector field on $X$. This was done in two cases, for a holomorphic vector field (when $X$ is a complex manifold), and for a vector field preserving a Riemannian metric on $X$. The second case was later better understood in the setting of equivariant cohomology [2]: one can introduce an equivariant de Rham complex, and the integral of any cocycle can be expressed in terms of its restrictions to the fixed points of the group action (which is an $S^{1}$ action for the case of a vector field preserving a Riemannian metric).

The analogy between the two cases, the holomorphic and the Riemannian one, is not complete. However, it is possible to define some kind of "formally equivariant" Dolbeault complex and derive from it localization formulas, see e.g. [16]. Our goal in this paper is to propose a comprehensive approach to holomorphic equivariant cohomology. We start from the holomorphic Atiyah algebroids $\mathscr{D}_{\mathscr{E}}$ associated to a holomorphic vector bundle $\mathscr{E}$ on a complex manifold $X$, and assuming that there is a vector field on $X$ that may be lifted to a section of $\mathscr{D}_{\mathscr{E}}$, we introduce a holomorphic equivariant complex and prove a related localization formula (Section 2). When $\mathscr{E}=\{0\}$ this cohomology essentially reduces to K. Liu's holomorphic equivariant cohomology [16]. By considering a twist by an effective line bundle, we can also prove a general localization formula related to the action of a meromorphic vector field.

Our localization formula contains the Baum-Bott formula [3], Feng-Ma's localization formula [11, and the Carrell-Liebermann residue formula [9, 8] as special cases. In all instances our approach produces significant simplifications with respect to the original proofs. Let us explain in which sense these formulas are special cases of ours. One of the ingredients of the theory is a (in general, meromorphic) vector field $V$ on $X$, which is lifted to a first-order differential operator acting on a holomorphic vector bundle $\mathscr{E}$. This lift is in general obstructed, therefore one needs to assume its existence. This is what we do, and is one of the assumptions of the Carrell-Liebermann formula; our formula is more general than the latter because we compute the integral of any cocycle in a certain cohomology complex that we associate with $V$ and $\mathscr{E}$, while the Carrell-Liebermann formula computes the integral of a polynomial in the Atiyah class of $\mathscr{E}$.

A special situation arises when $\mathscr{E}$ is the holomorphic tangent bundle $\Theta_{X}$. If the vector field $V$ is holomorphic, it always has a lift to a differential operator on $\Theta_{X}$, namely, the 
Lie derivative $£_{X}$. Thus one gets a generalization of Bott formula [5], or, if one integrates a polynomial in the Chern classes of $X$, Bott's formula itself.

If $V$ is meromorphic, i.e., it is a section of $\Theta_{X} \otimes \mathscr{L}$ for some effective line bundle $\mathscr{L}$, in general it does not lift. There are two ways out of this problem. Either one just treats the special case when the obstruction vanishes; this produces a particular case of our localization formula (4.1). Or, one realizes that even if $V$ does not lift, it actually defines a residue (as noticed by Carrell [7], see also section [4.5 in this paper). However, this residue does not compute a characteristic number of the tangent bundle $\Theta_{X}$, but rather a characteristic number of the virtual bundle $\Theta_{X}-\mathscr{L}^{*}$. Thus one obtains Baum-Bott's formula.

Further investigations along this line would naturally lead to consider generalizations of the holomorphic Lefschetz formulas and applications to Courant algebroids, e.g., in connection to generalized complex geometry (cf. [15]).

Acknowledgments. This paper was drafted while the first author was visiting IMPA in Rio de Janeiro, and was finalized while he was holding a visiting position at IHES in Paris. He gratefully acknowledges support and hospitality from both institutions. The second author thanks LPTM of the Cergy-Pontoise University for hospitality during his CNRS delegation. We thank Professors Yvette Kosmann-Schwarzbach and Alexei Rosly for their interest in this work.

\section{TWISTED HOLOMORPHIC EQUIVARIANT COHOMOLOGY}

Let $X$ be an $n$-dimensional compact complex manifold. We shall denote by $\Theta_{X}$ its holomorphic tangent bundle and by $T_{X}$ its tangent bundle when $X$ is regarded as a $2 n$ dimensional smooth differentiable manifold. $\Omega_{X}^{i}$ will denote the bundle of holomorphic $i$-forms on $X$, while $\Omega_{X, \mathbb{C}}^{i}$ will denote the bundle of complex-valued smooth $i$-forms, and $\Omega_{X}^{p, q}$ the bundle of forms of type $(p, q)$. (In general, we shall not use a different notation for a bundle and its sheaf of sections.) The symbol $\Gamma$ will denote global sections.

We shall not use any Lie algebroid theory here but one should note that our constructions are quite heavily motivated and suggested by that theory [10, 14, 6].

2.1. The Atiyah algebroid. If $\mathscr{E}$ is a (rank $r$ ) holomorphic vector bundle on $X$ we shall denote by $\mathscr{D}_{\mathscr{E}}$ the bundle of first order differential operators on $\mathscr{E}$ with scalar symbol. $\mathscr{D}_{\mathscr{E}}$ sits inside an exact sequence of sheaves of $\mathscr{O}_{X}$-modules

$$
0 \rightarrow \operatorname{End}(\mathscr{E}) \rightarrow \mathscr{D}_{\mathscr{E}} \stackrel{\sigma}{\rightarrow} \Theta_{X} \rightarrow 0
$$


where $\sigma$ is the symbol map. $\mathscr{D}_{\mathscr{E}}$ is actually an example of a holomorphic Lie algebroid [10, 14, with bracket defined on its sections by the commutator of differential operators, and the symbol map $\sigma$ playing the role of anchor.

The class in $a(\mathscr{E}) \in \operatorname{Ext}^{1}\left(\Theta_{X}, \operatorname{End}(\mathscr{E})\right)$ defining the extension (11) is called the Atiyah class of $\mathscr{E}$, and it is the obstruction to the existence of holomorphic connections on $\mathscr{E}$ [1].

We shall assume throughout that a global holomorphic vector field $V$ on $X$ has been fixed, which admits a lift $\tilde{V}$ in $\Gamma\left(\mathscr{D}_{\mathscr{E}}\right)$. The pair $(\mathscr{E}, \tilde{V})$ is called an equivariant holomorphic vector bundle.

2.2. The cohomology complex. We want to define a "holomorphic equivariant" cohomology complex associated with the Atiyah algebroid $\mathscr{D}_{\mathscr{E}}$. We set

$$
\begin{gathered}
D_{\mathscr{E}}=\left[\mathscr{C}_{X}^{\infty} \otimes_{\mathscr{O}_{X}} \mathscr{D}_{\mathscr{E}}\right] \oplus T_{X}^{0,1} \\
Q_{\mathscr{E}}^{p, q}=\Lambda^{p} \mathscr{D}_{\mathscr{E}}^{*} \otimes_{\mathscr{O}_{X}} \Omega_{X}^{0, q} \\
Q_{\mathscr{E}}^{k}=\Lambda^{k} D_{\mathscr{E}}^{*}=\bigoplus_{p+q=k} Q_{\mathscr{E}}^{p, q}
\end{gathered}
$$

Note that the line bundle $\operatorname{det}\left(D_{\mathscr{E}}\right)$ is isomorphic to the determinant of the complexified tangent bundle $T_{X} \otimes \mathbb{C}$. Moreover, combining the symbol map with the inner product one defines a morphism

$$
q: \Lambda^{k} D_{\mathscr{E}}^{*} \otimes \operatorname{det}\left(D_{\mathscr{E}}\right) \rightarrow \Lambda^{2 n-k+r^{2}}\left(T_{X} \otimes \mathbb{C}\right) .
$$

We define a morphism $p: Q_{\mathscr{E}}^{k} \rightarrow \Omega_{X, \mathbb{C}}^{k-r^{2}}$ by setting 1

$$
\left.p(\psi)=(-1)^{k} q(\psi \otimes \alpha)\right\lrcorner \eta
$$

if $\psi$ is a section of $\Lambda^{k} D_{\mathscr{E}}^{*}$, and $\alpha \in \Gamma\left(\operatorname{det}\left(D_{\mathscr{E}}\right)\right)$ and $\eta$ in $\Omega_{X, \mathbb{C}}^{2 n}(X)$ are such that $\eta(\alpha)=1$. This map is an isomorphism when $\mathscr{E}=\{0\}$. By using this map we can integrate sections of $Q_{\mathscr{E}}^{\bullet}$, i.e., if $\gamma \in Q_{\mathscr{E}}^{\bullet}(X)$, and $X$ is compact, by $\int_{X} \gamma$ we mean $\int_{X} p(\gamma)$.

We define an "equivariant" complex

$$
\mathfrak{Q}_{\mathscr{E}}^{\bullet}=\mathbb{C}[t] \otimes_{\mathbb{C}} Q_{\mathscr{E}}^{\bullet}(X)
$$

with the usual equivariant grading

$$
\operatorname{deg}(\mathscr{P} \otimes \beta)=2 \operatorname{deg}(\mathscr{P})+\operatorname{deg} \beta
$$

\footnotetext{
${ }^{1}$ Here we use the symbol $\lrcorner$ to denote inner product, however for typographical reasons later on we shall also use the symbol $\imath$.
} 
if $\mathscr{P}$ is a monomial in $t$ and $\beta \in Q_{\mathscr{E}}^{\bullet}$. We also define a differential

$$
\tilde{\delta}_{V}=\bar{\partial}_{\mathscr{D} \mathscr{E}}-t \imath_{\tilde{V}}
$$

where $\bar{\partial}_{\mathscr{D}_{\mathscr{E}}}$ is any of the Cauchy-Riemann operators of the holomorphic bundles $\Lambda^{k} \mathscr{D}_{\mathscr{E}}{ }^{*}$. We have $\tilde{\delta}_{V}: \mathfrak{Q}_{\mathscr{E}}^{\bullet} \rightarrow \mathfrak{Q}_{\mathscr{E}}^{\bullet+1}$, and an easy computation shows that $\tilde{\delta}_{V}^{2}=0$, so that $\left(\mathfrak{Q}_{\mathscr{E}}^{\bullet}, \tilde{\delta}_{V}\right)$ is a cohomology complex. We denote its cohomology by $\mathfrak{H}_{\tilde{V}}^{\bullet}(\mathscr{E})$.

There is a relation between the complex $\mathfrak{Q}_{0}^{\bullet}$ that one obtains by setting $\mathscr{E}=\{0\}$ in $\mathfrak{Q}_{\mathscr{E}}^{\bullet}$ and Liu's holomorphic equivariant de Rham complex [16]. Liu's complex is defined letting

$$
A^{(k)}=\bigoplus_{q-p=k} \Omega^{p, q}(X)
$$

with a differential

$$
\delta_{t}=\bar{\partial}-t \imath_{V}
$$

for some value of $t$. One has, for every $t$, cohomology complexes $\left(A^{(k)}, \delta_{t}\right)$, where the index $k$ ranges from $-n$ to $n$. Liu shows that the corresponding cohomology groups $H_{t}^{(k)}(X)$ are independent of $t$, provided $t \neq 0$. We shall denote them by $H_{\mathrm{Liu}}^{\bullet}(X)$. An explicit computation shows the following relation. Let us denote by $\mathfrak{H}_{V}^{\bullet}(X)$ the cohomology groups $\mathfrak{H}_{\tilde{V}}^{\bullet}(\mathscr{E})$ corresponding to the case $\mathscr{E}=\{0\}$. Moreover, let $\tilde{\mathfrak{H}}_{V}^{2 n+k}(X)$ be the subspace of $\mathfrak{H}_{V}^{2 n+k}(X)$ formed by classes of cocycles in the subspace $\oplus_{p}\left[\mathbb{C}[t] \otimes \Omega^{p, p+k}(X)\right]$ of $\oplus_{j} \mathfrak{Q}_{0}^{j}$.

Proposition 2.1. For every $k=-n, \ldots, n$, and every $t^{*} \in \mathbb{C}^{*}$, the cohomology group $H_{\text {Liu }}^{(k)}(X)$ is isomorphic as a $\mathbb{C}$-vector space to the subspace of $\tilde{\mathfrak{H}}_{V}^{2 n+k}(X)$ obtained by setting $t=t^{*}$.

Proof. Let us realize $H_{\mathrm{Liu}}^{(k)}(X)$ as $H_{1}^{(k)}(X)$. A class $[\omega]$ in this group is represented by an element

$$
\omega=\sum_{p=0}^{n} \omega^{p, p+k}
$$

(where $\left.\omega^{p, p+k} \in \Omega^{p, p+k}(X)\right)$ satisfying $\left(\bar{\partial}-\imath_{V}\right) \omega=0$, i.e.,

$$
\bar{\partial} \omega^{p, p+k}=\imath_{V} \omega^{p+1, p+k+1} \quad \text { for } \quad p=0, \ldots, n .
$$

If we define the element in $\mathfrak{Q}_{0}^{\bullet}$

$$
\xi=\sum_{p=0}^{n} t^{n-p} \omega^{p, p+k}
$$

then the condition (4) is equivalent to $\tilde{\delta}_{V} \xi=0$. Thus we obtain a class in $\tilde{\mathfrak{H}}_{V}^{2 n+k}(X)$. Conversely, given a cocycle representing a class in this space, the previous computation 
shows that evaluating it at $t=1$ we get a class in $H_{1}^{(k)}(X)$. The same is true for any $t^{*} \in \mathbb{C}^{*}$.

In particular $H_{\text {Liu }}^{(0)}(X)$ is isomorphic to the subspace of $\oplus_{j} \mathfrak{H}_{V}^{j}(X)$ generated over $\mathbb{C}[t]$ by the classes in $\oplus_{p} \Omega^{p, p}(X)$.

\section{LOCALIZATION}

We turn now to the construction of a localization formula for the holomorphic equivariant cohomology $\mathfrak{H}_{\tilde{V}}^{\bullet}(\mathscr{E})$ we introduced in Section 2.2. Since we want to cover also the case when the vector field $V$ has nonsimple zeroes we need to introduce the notion of Grothendieck residue [13, 8].

3.1. The Grothendieck residue. We recall here the definition as given in [8]. Let us start from the situation where we have a line bundle $\mathscr{L}$ on $\mathbb{C}^{n}$, and $n$ sections $a_{1}, \ldots, a_{n}$ of $\mathscr{L}$ that have a common isolated zero at 0 . Let $s$ be a section of $\mathscr{L}^{n} \otimes \Omega_{\mathbb{C}^{n}}^{n}$, and let $D$ be a disc in $\mathbb{C}$, centred at 0 , such that $D^{n}$ does not contain zeroes of $a_{1}, \ldots, a_{n}$ other than 0 , and the product $a_{1} \cdots a_{n}$ does not vanish on $\partial D \times \cdots \times \partial D$. Then one sets

$$
\operatorname{Res}\left(\begin{array}{c}
s \\
a_{1} \ldots a_{n}
\end{array}\right)=\frac{1}{(2 \pi i)^{n}} \int_{\partial D \times \cdots \times \partial D} \frac{s}{a_{1} \cdots a_{n}}
$$

Now, given a line bundle $\mathscr{L}$ on $X$, let $V$ be a global section of $\Theta_{X} \otimes \mathscr{L}$ that has isolated, but possibly degenerate, zeroes. If $x_{0}$ is one of such zeroes, and $s$ is a section of $\mathscr{L}^{n} \otimes \Omega_{X}^{n}$ on a neighbourhood of $x_{0}$, let us choose holomorphic coordinates $\left(z_{1}, \ldots, z_{n}\right)$ centred in $x_{0}$, and write $V=\sum_{i} a_{i} \frac{\partial}{\partial z_{i}}$ locally around $x_{0}$. We can define

$$
\operatorname{Res}_{V, x_{0}}(s)=\operatorname{Res}\left(\begin{array}{c}
s \\
a_{1} \ldots a_{n}
\end{array}\right) .
$$

In the particular case when the zero of $V$ at $x_{0}$ is nondegenerate, so that the Jacobian determinant $J\left(a_{1} \ldots a_{n}\right)_{x_{0}}$ of the partial derivatives of the components $a_{i}$ at $x_{0}$ does not vanish, one can write

$$
\operatorname{Res}_{V, x_{0}}(s)=\frac{s\left(x_{0}\right)}{J\left(a_{1} \ldots a_{n}\right)_{x_{0}}} .
$$

An algorithm to get an explicit expression for the Grothendieck residue in the general case (i.e., when $V$ has degenerate zeroes) is given in [3].

The residue (66) is independent of the choices of the coordinates, and defines a morphism

$$
\operatorname{Res}_{V, Z}: H^{0}\left(Z, \mathscr{L}^{n}\right) \rightarrow \mathbb{C}
$$


where $Z$ is the closed 0 -dimensional subscheme of $X$ given by the zeroes of $V$ (more precisely, $Z$ is the subscheme associated with the sheaf of ideals $\left.\mathscr{I}_{Z}=\imath_{V}\left(\Omega_{X}^{1} \otimes \mathscr{L}^{*}\right) \subset \mathscr{O}_{X}\right)$.

The previous discussion implies the following result.

Lemma 3.1. If $V$ has isolated nondegenerate zeroes $\left\{x_{j}\right\}$, one has

$$
\operatorname{Res}_{V, Z}(s)=\sum_{j} \frac{s\left(x_{j}\right)}{\operatorname{det} \mathbb{L}_{V, j}}
$$

where

$$
\mathbb{L}_{V, j}: \Theta_{X, x_{j}} \rightarrow\left(\Theta_{X} \otimes \mathscr{L}\right)_{x_{j}}
$$

is the linear transformation defined as $\mathbb{L}_{V, j}(u)=[V, u]$.

Note that $\operatorname{det} \mathbb{L}_{V, j} \in \mathscr{L}_{x_{j}}^{n}$.

3.2. The localization formula. We consider at first a localization formula in the case when the line bundle involved in the definition of the Grothendieck residue is trivial, $\mathscr{L}=\mathscr{O}_{X}$.

Theorem 3.2. Let $X$ be an $n$-dimensional compact complex manifold, $\mathscr{E}$ a holomorphic vector bundle on $X$, and $V$ a holomorphic vector field on $X$, which lifts to a section of $\mathscr{D}_{\mathscr{E}}$, and has isolated zeroes. If $\gamma \in \mathfrak{Q}_{\mathscr{E}}^{\bullet}$ is such that $\tilde{\delta}_{V} \gamma=0$, we have

$$
\int_{X} \gamma(t)=\left(\frac{2 \pi i}{t}\right)^{n} \operatorname{Res}_{V, Z}\left(p(\gamma)_{0}(t)\right) .
$$

where $Z$ is the scheme of the zeroes of $V$.

Remark 3.3. If the zeroes $\left\{x_{j}\right\}$ are nondegenerate, in view of Lemma 3.1 the localization formula may be written as

$$
\int_{X} \gamma(t)=(2 \pi i)^{n} \sum_{j} \frac{p(\gamma)_{0}\left(x_{j}\right)(t)}{t^{n} \operatorname{det} \mathbb{L}_{V, j}}
$$

where $\mathbb{L}_{V, j}: \Theta_{X, x_{j}} \rightarrow \Theta_{X, x_{j}}$. In this form this formula is basically equivalent to Feng-Ma's localization formula [11].

Proof. The proof of the localization formula in equivariant cohomology as given in [4] may be adapted to provide an easy proof of formula (8) .

The map $p: Q_{\mathscr{E}}^{k} \rightarrow \Omega_{X, \mathbb{C}}^{k-r^{2}}$ may be written - with respect to the decomposition (2) as $p=\sum_{i+j=k} p_{i} \otimes \imath_{j}$, where $p_{i}: \mathscr{C}_{X}^{\infty} \otimes \Lambda^{i} \mathscr{D}_{\mathscr{E}}{ }^{*} \rightarrow \Omega_{X}^{i-r^{2}, 0}$, and $\imath_{j}: \Omega_{X}^{0, j} \rightarrow \Omega_{X}^{0, j}$ is the identity map. This implies the identity

$$
p \circ \bar{\partial}_{D_{\mathscr{E}}}=\bar{\partial} \circ p
$$


This reduces the proof of the localization formula to the case $\mathscr{E}=\{0\}$. Let us denote by $\delta_{V}=\bar{\partial}-t \imath_{V}$ the "holomorphic equivariant" differential for the complex $\mathfrak{Q}_{0}^{\bullet}=\mathbb{C}[t] \otimes \Omega_{X, \mathbb{C}}^{\bullet}$.

Choose an hermitian metric $g$ on $X$, and denoting by $\bar{g}: T_{X}^{0,1} \rightarrow \Omega_{X}^{1,0}$ the corresponding homomorphism, let $\theta=\bar{g}(\bar{V}) /\|V\|^{2}$ (so $\theta$ is of type $(1,0)$ ). Using the injection $\Omega_{X, \mathbb{C}}^{1} \rightarrow D_{\mathscr{E}}^{*}$, $\theta$ may be regarded as a cochain in $\mathfrak{Q}_{\mathscr{E}}^{1}$. Note that $\left(\delta_{V} \theta\right)_{0}=-t$ so that $\delta_{V} \theta$ is invertible in the ring of differential forms away from the zeroes of $V$ if $t \neq 0$, and one has

$$
\delta_{V}\left(\frac{\theta}{\delta_{V} \theta}\right)=1
$$

We set

$$
\alpha=\frac{\theta}{\delta_{V} \theta}, \quad \tilde{\alpha}=\alpha \wedge \gamma
$$

so that $\delta_{V} \tilde{\alpha}=\gamma$ (again, away from the zeroes of $V$ ). If we set

$$
\gamma=\sum_{k=0}^{n} t^{k} \gamma_{2 n-k}, \quad \alpha=\sum_{k=0}^{n-1} \alpha_{2 k+1}
$$

with

$$
\alpha_{2 k+1}=\theta\left(\frac{1}{\tilde{\delta} \theta}\right)_{(2 k)}=-(-t)^{-k-1} \theta \wedge(\bar{\partial} \theta)^{k}
$$

then away from the zeroes of $V$ one has

$$
\gamma_{2 n}=\bar{\partial} w
$$

with

$$
w=-\sum_{k=0}^{n} \gamma_{2 n-2 k} \wedge \theta \wedge(\bar{\partial} \theta)^{k-1} .
$$

Note that $p(\gamma)_{0}=\gamma_{0}$. Denoting by $x_{j}$ the zeroes of $V$, let $B_{j}(\epsilon)$ be a ball of radius $\epsilon$ (with respect to the hermitian metric we chose) with center $x_{j}$. Then by Stokes theorem and equation (10)

$$
\int_{X} \gamma=-\sum_{j} \lim _{\epsilon \rightarrow 0} \int_{\partial B_{j}(\epsilon)} w=-\sum_{j} \lim _{\epsilon \rightarrow 0} \int_{B_{j}(\epsilon)} \bar{\partial} w=\sum_{j} \lim _{\epsilon \rightarrow 0} \int_{B_{j}(\epsilon)} \gamma_{0}(\bar{\partial} \theta)^{n} .
$$

Let $Z_{j}$ be the component of $Z$ supported on $x_{j}$. Since we may replace each ball $B_{j}(\epsilon)$ with a polydisk of radius, say, $\epsilon$, comparing with the definition (5) of the Grothendieck residue we get

$$
\lim _{\epsilon \rightarrow 0} \int_{B_{j}(\epsilon)} \gamma_{0}(\bar{\partial} \theta)^{n}=(2 \pi i)^{n} \operatorname{Res}_{V, Z_{j}}\left(\gamma_{0}\right)
$$

and therefore we obtain the expression of the right-hand side of the localization formula. The reader may also compare with the proof of formula (9) in [7]. 
Remark 3.4. If we take $\mathscr{E}=\{0\}$ and consider only classes $\gamma$ in $\mathbb{C}[t] \otimes\left[\oplus_{p} \Omega^{p, p}(X)\right]$, this localization formula reduces to Liu's formula [16, Thm. 1.6].

Example 3.5. If $\mathscr{E}=\mathscr{O}_{X}$, the exact sequence (1) splits, and one has

$$
Q_{\mathscr{O}_{X}}^{k}=\Omega_{X, \mathbb{C}}^{k} \oplus \Omega_{X, \mathbb{C}}^{k-1}
$$

The morphism $p$ maps $Q_{\mathscr{O}_{X}}^{k}$ to $\Omega_{X, \mathbb{C}}^{k-1}$. An element in $\mathfrak{Q}_{\mathscr{O}_{X}}^{2 n+1}$ (where $n=\operatorname{dim}_{\mathbb{C}} X$ ) has the form

$$
\sum_{k=0}^{n}\left(\omega_{2 n+1-2 k}+\eta_{2 n+1-2 k}\right) t^{k}
$$

where $\omega_{k} \in \Omega_{X, \mathbb{C}}^{k}(X)$ and $\eta_{k} \in \Omega_{X, \mathbb{C}}^{k-1}(X)$.

Let $V$ be a holomorphic vector field on $X$ with isolated nondegenerate zeroes. If $\omega_{2} \in$ $\Omega_{X, \mathbb{C}}^{2}(X)$ and $f \in \mathscr{C}^{\infty}(X)$ are such that $\bar{\partial} \omega_{2}=0, \iota_{V} \omega_{2}=\bar{\partial} f$, then $\omega=\left(\omega_{2}+t f\right)^{n} \in \mathfrak{Q}_{\mathscr{O}_{X}}^{2 n+1}$ is a cocycle, i.e., $\hat{\delta}_{V} \omega=0$. The localization formula (8) gives

$$
\int_{X}\left(\omega_{2}\right)^{n}=(-2 \pi i)^{n} \sum_{\ell} \frac{f\left(x_{\ell}\right)}{J_{\ell}}
$$

where $x_{\ell}$ are the zeroes of $V$, and $J_{\ell}$ are the Jacobian determinants of the components of $V$ at those zeroes. Equation (11) is a kind of complex Duistermaat-Heckman formula (but $\omega_{2}$ need not be nondegenerate).

The existence of the holomorphic vector field $V$ with isolated zeroes, and of nontrivial $\omega_{2}, f$ satisfying $\iota_{V} \omega_{2}=\bar{\partial} f$, puts conditions on the variety $X$. If $X=\mathbb{P}^{1}$, such a vector field obviously exists, and a simple check, using the Fredholm alternative for the Laplacian on functions on $\mathbb{P}^{1}$, shows that for any choice of $\omega_{2}$, there exists a function $f$ satisfying $\iota_{V} \omega_{2}=\bar{\partial} f .\left(\bar{\partial} \omega_{2}=0\right.$ is automatic. $)$

3.3. Moment maps. We develop some further techniques, in particular we introduce an appropriate notion of moment map. Let $K$ be the curvature of the Chern connection $\nabla$ of the pair $(\mathscr{E}, h)$ (where $h$ is an hermitian metric on $\mathscr{E}$ ), i.e., the unique connection on $\mathscr{E}$ which is compatible both with the holomorphic structure of $\mathscr{E}$ and the metric $h$. Let $V$ be a holomorphic vector field on $X$, with isolated zeroes, and let $\tilde{V}$ be a lift of $V$. The moment map $\mu$ is the $C^{\infty}$ endomorphism of $\mathscr{E}$ given by

$$
\mu=\tilde{V}-\nabla_{V} .
$$

Lemma 3.6. The moment map $\mu$ enjoys the following properties:

(i) $\bar{\partial}_{\operatorname{End}(\mathscr{E})} \mu=\imath_{V} K$, where $K$ is the curvature of $\nabla$; 
(ii) $\mu\left(x_{j}\right)=\mathbb{L}_{\tilde{V}, j}$ for all zeroes $x_{j}$ of $V$, where $\mathbb{L}_{\tilde{V}, j}: \mathscr{E}_{x_{j}} \rightarrow \mathscr{E}_{x_{j}}$ is the endomorphism induced by the differential operator $\tilde{V}$ (note that at the zeroes $x_{j}$ of $V$, the differential operator $\tilde{V}$ has degree 0 ).

Proof. The first claim is proved as in [5] by the following chain of identities. Let $u$ be a vector field of type $(0,1)$ on $X$, and $s$ a section of $\mathscr{E}$.

$$
\begin{aligned}
\left\langle\left(\bar{\partial}_{E n d(\mathscr{E})} \mu\right)(s), u\right\rangle & =\left\langle\bar{\partial}_{\mathscr{E}}(\mu(s)), u\right\rangle=-\nabla_{u} \nabla_{V} s \\
& =\nabla_{V} \nabla_{u} s-\nabla_{u} \nabla_{V} s-\nabla_{[V, u]} s=\langle K(s), V \wedge u\rangle \\
& =\left\langle\iota_{V} K(s), u\right\rangle .
\end{aligned}
$$

The second statement is evident.

We define the equivariant curvature of the Chern connection as $\tilde{K}=K+t \mu$. By using the connection $\nabla$ to split the exact sequence

$$
0 \rightarrow \operatorname{End}(\mathscr{E}) \otimes \mathscr{C}_{X}^{\infty} \rightarrow D_{\mathscr{E}} \rightarrow T_{X} \otimes \mathbb{C} \rightarrow 0
$$

we may regard $\tilde{K}$ as an element in $\mathfrak{Q}_{\mathscr{E}}^{3}$.

Lemma 3.7. For every $m \geq 0$, the the cochain $\operatorname{tr}\left(\tilde{K}^{m}\right) \in \mathfrak{Q}_{\mathscr{E}}^{2 m}$ is closed, i.e., $\tilde{\delta}_{V} \operatorname{tr}\left(\tilde{K}^{m}\right)=$ 0 .

Proof. After splitting the exact sequence (13), the complex $\mathfrak{Q}_{\mathscr{E}}^{\bullet}$ acquires a bigrading; let $\Omega_{X, \mathbb{C}}^{1}$ have bidegree $(1,0)$, and $\operatorname{End}(\mathscr{E})$ bidegree $(0,1)$. Then $\operatorname{tr}\left(\tilde{K}^{m}\right)$ has bidegree $(2 m, 0)$, while $\bar{\partial}_{\mathscr{D} \mathscr{E}}$ has bidegree $(1,0)$, and $\imath_{\tilde{V}}$ has a piece of bidegree $(-1,0)$ (which is basically $\imath_{V}$ ) and a piece of bidegree $(0,-1)$. Then one has

$$
\begin{aligned}
\tilde{\delta}_{V} \operatorname{tr}\left(\tilde{K}^{m}\right)=\left(\bar{\partial}_{\mathscr{D}_{\mathscr{E}}}-t \imath_{\tilde{V}}\right) \operatorname{tr}\left(\tilde{K}^{m}\right)=\left(\bar{\partial}_{\mathscr{D}_{\mathscr{E}}}-t \imath_{V}\right) \operatorname{tr}\left(\tilde{K}^{m}\right)= \\
m \operatorname{tr}\left(\tilde{K}^{m-1}\left(\bar{\partial}_{\mathscr{D}_{\mathscr{E}}}-\imath_{V}\right) \tilde{K}\right)=m \operatorname{tr}\left(\tilde{K}^{m-1}\left(\bar{\partial}_{\mathscr{D}_{\mathscr{E}}} K+t \bar{\partial}_{\mathscr{D}_{\mathscr{E}}} \mu-t \imath_{V} K\right)\right)=0
\end{aligned}
$$

the equality to zero of the last expression being due to the Bianchi identities and the definition of the moment map.

Example 3.8. Assume that $\mathscr{E}$ is a line bundle $\mathscr{M}$, and define

$$
\gamma(t)=\left(\frac{i}{2 \pi} \tilde{K}\right)^{n}
$$

where $n=\operatorname{dim} X$. Then $\gamma$ is a cocycle in $\mathfrak{Q}_{\mathscr{E}}^{\bullet}$, and moreover we have

$$
p(\gamma)_{0}(t)=\left(\frac{i t}{2 \pi} \mu\right)^{n} \text {. }
$$


Assume moreover that $V$ has isolated nondegenerate zeroes. Theorem 3.2 yields

$$
\int_{X} c_{1}(\mathscr{M})^{n}=\sum_{j} \frac{\left(c_{j}\right)^{n}}{\operatorname{det}\left(\mathbb{L}_{V, j}\right)} .
$$

Here $c_{1}(\mathscr{M})$ is a $(1,1)$-form on $X$ representing the first Chern class of $\mathscr{M}$, and $c_{j}$ are complex numbers such that $\mathbb{L}_{\tilde{V}, j}(z)=c_{j} z$. This equation is a special case of the CarrellLiebermann localization formula [9, 8]; indeed, in the next Section, we shall see that the formula (8) implies the Carrell-Liebermann formula.

If $\mathscr{M}=\mathscr{O}_{X}$, we have $a\left(\mathscr{O}_{X}\right)=0$ and the exact sequence defining the Atiyah algebroid $\mathscr{D}_{\mathscr{O}_{X}}$ splits. Every holomorphic vector field $V$ on $X$ lifts to a section $\tilde{V}$ of $\mathscr{D}_{\mathscr{O}_{X}}$ of the form $c+V$, with $c$ a constant. Formula (14) yields the well-known identity [5]

$$
\sum_{j} \frac{1}{\operatorname{det}\left(\mathbb{L}_{V, j}\right)}=0 \text {. }
$$

3.4. Bott's formula. To recover Bott's classical formula we set $\mathscr{E}=\Theta_{X}$. Any holomorphic vector field $V$ on $X$ has a lift to a differential operator $\tilde{V}$ on $\Theta_{X}$, which is $V$ itself acting as s Lie derivative. At the zeroes $x_{j}$ of $V$, this defines an endomorphism $\mathbb{L}_{\tilde{V}, x_{j}}:\left(\Theta_{X}\right)_{x_{j}} \rightarrow\left(\Theta_{X}\right)_{x_{j}}$. Moreover, in this case $p: Q_{\mathscr{E}}^{2 n+r^{2}}=Q_{\Theta_{X}}^{2 n+n^{2}} \rightarrow \Omega_{X, \mathbb{C}}^{2 n}$ is an isomorphism. We assume that the zeroes of $V$ are nondegenerate.

Let $P_{k}$ be the $k$-th invariant elementary polynomial on the Lie algebra $\operatorname{gl}(n, \mathbb{C})$, where $n=\operatorname{dim} X$. We use the normalization of [12]. We define the equivariant Chern classes of $X$ as

$$
\tilde{c}_{k}(X)=P_{k}\left(\frac{i}{2 \pi} \tilde{K}\right), \quad k=1, \ldots, n
$$

and the equivariant Chern classes of the endomorphisms $\mathbb{L}_{V, j}: \Theta_{X, x_{j}} \rightarrow \Theta_{X, x_{j}}$ as

$$
\tilde{c}_{k}\left(x_{j}\right)=P_{k}\left(\frac{i}{2 \pi} \tilde{\mu}\left(x_{j}\right)\right), \quad k=1, \ldots, n .
$$

Note that $\tilde{c}_{n}\left(x_{j}\right)=\operatorname{det}\left(\mathbb{L}_{V, j}\right)$. Let $\Phi$ be a polynomial in $n$ variables whose degree in the $i$-th variable is $m_{i}$, and such that $\sum_{i=1}^{n} i m_{i}=n$. By Lemma 3.7, the quantity

$$
\Phi\left(\tilde{c}_{1}(X), \ldots, \tilde{c}_{n}(X)\right)
$$

is a cocycle in $\mathfrak{Q}_{\Theta_{X}}^{\bullet}$. The localization formula (8) in this case reads

$$
\int_{X} \Phi\left(c_{1}(X), \ldots, c_{n}(X)\right)=\sum_{j} \frac{\Phi\left(\tilde{c}_{1}\left(x_{j}\right), \ldots, \tilde{c}_{n}\left(x_{j}\right)\right)}{\tilde{c}_{n}\left(x_{j}\right)},
$$

i.e., we recover Bott's formula. (One uses the formula in Lemma 3.1; note that the determinant in the denominator of the residue is just the Chern class $\tilde{c}_{n}\left(x_{j}\right)$.) 


\section{THE TWISTED CASE}

One can also write a localization formula in the case when $V$ is a global section of $\Theta_{X} \otimes \mathscr{L}$, where $\mathscr{L}$ is a holomorphic line bundle. The advantage in making such a twist is that if $X$ is projective, then by Serre theorem one can always choose $\mathscr{L}$ so that there exist sections of $H^{0}\left(X, \Theta_{X} \otimes \mathscr{L}\right)$ that may be lifted to a differential operator $\mathscr{E} \rightarrow \mathscr{E} \otimes \mathscr{L}$ [9, 8].

If $\mathscr{L}$ is an effective line bundle, i.e., $\mathscr{L} \simeq \mathscr{O}_{X}(D)$ for an effective divisor $D$ in $X$, the localization formula we shall obtain contains Baum-Bott's formula for meromorphic vector fields [3] and Carell-Liebermann's formula [9] as special cases.

4.1. A new cohomology complex. We define a new cohomology complex by setting 2

$$
\widehat{Q}_{\mathscr{E}}^{k}=\bigoplus_{p+q=k} \widehat{Q}_{\mathscr{E}}^{p, q}, \quad \widehat{Q}_{\mathscr{E}}^{p, q}=\Lambda^{p} \mathscr{D}_{\mathscr{E}}{ }^{*} \otimes_{\mathscr{O}_{X}} \mathscr{L}^{-p+n+r^{2}} \otimes_{\mathscr{O}_{X}} \Omega_{X}^{0, q}
$$

and

$$
\widehat{\mathfrak{Q}}_{\mathscr{E}}^{\bullet}=\mathbb{C}[t] \otimes_{\mathbb{C}} \widehat{Q}_{\mathscr{E}}^{\bullet}(X),
$$

with a grading and differential defined as in the previous case. Moreover, we may define a map

$$
\begin{aligned}
\hat{q}: \bigoplus_{p+q=k} \Lambda^{p} \mathscr{D}_{\mathscr{E}}^{*} \otimes_{\mathscr{O}_{X}} \mathscr{L}^{-p+n+r^{2}} \otimes_{\mathscr{O}_{X}} \operatorname{det} & \mathscr{D}_{\mathscr{E}} \otimes_{\mathscr{O}_{X}} \Omega_{X}^{0, q} \\
& \rightarrow \bigoplus_{p+q=k} \Lambda^{-p+n+r^{2}} \Theta_{X} \otimes_{\mathscr{O}_{X}} \mathscr{L}^{-p+n+r^{2}} \otimes_{\mathscr{O}_{X}} \Omega_{X}^{0, q}
\end{aligned}
$$

by setting

$$
\hat{q}(\phi \otimes s \otimes u \otimes \omega)=\sigma(\phi\rfloor u) \otimes s \otimes \omega
$$

where $\sigma$ is the anchor $\mathscr{D}_{\mathscr{E}} \rightarrow \Theta_{X}$. We also define

$$
\hat{p}: \widehat{Q}_{\mathscr{E}}^{k} \rightarrow \bigoplus_{p+q=k} \mathscr{L}^{-p+n+r^{2}} \otimes_{\mathscr{O}_{X}} \Omega_{X}^{p-r^{2}, q}
$$

by letting $\left.\hat{p}(\psi)=(-1)^{k} \hat{q}(\psi \otimes \alpha)\right\rfloor \eta$, where $\alpha \in \Gamma\left(\mathscr{C}_{X}^{\infty} \otimes_{\mathscr{O}_{X}} \operatorname{det}\left(\Theta_{X}\right)\right)$ and $\eta \in \Omega_{X}^{n, 0}(X)$ are such that $\eta(\alpha)=1$. Note that for $k=2 n+r^{2}$ one has $\hat{p}(\psi) \in \Omega_{X, \mathbb{C}}^{2 n}(X)$, and for $k=r^{2}$, $\hat{p}(\psi) \in \Gamma\left(\mathscr{C}_{X}^{\infty} \otimes_{\mathscr{O}_{X}} \mathscr{L}^{n}\right)$. Instead of the identity (9) we have now

$$
\hat{p} \circ \bar{\partial}_{\mathscr{D}}=(-1)^{k} \bar{\partial}_{\mathscr{L}} \circ \hat{p}
$$

where $\bar{\partial}_{\mathscr{D} \mathscr{E}}$ is now any of the $\bar{\partial}$-operators of the sheaves $\Lambda^{p} \mathscr{D}_{\mathscr{E}}{ }^{*} \otimes \mathscr{L}^{-p+n+r^{2}}$, and $\bar{\partial}_{\mathscr{L}}$ is the $\bar{\partial}$-operator of a suitable power of $\mathscr{L}$.

\footnotetext{
${ }^{2}$ For $\mathscr{E}=0$ this complex already appeared in [7].
} 
Again, we define a complex

$$
\widehat{\mathfrak{Q}}_{\mathscr{E}}^{\bullet}=\mathbb{C}[t] \otimes_{\mathbb{C}} \widehat{Q}_{\mathscr{E}}^{\bullet}(X)
$$

with a differential

$$
\hat{\delta}_{V}=\bar{\partial}_{\mathscr{D}}-t \imath_{\tilde{V}}
$$

4.2. Localization formula in the meromorphic case. We can now state and proof the localization formula for the meromorphic case.

Theorem 4.1. Let $X$ be an $n$-dimensional connected compact complex manifold, $\mathscr{E} a$ holomorphic vector bundle on $X, \mathscr{L}$ a holomorphic line bundle, and $V$ a global section of $\Theta_{X} \otimes \mathscr{L}$, which lifts to a section of $\mathscr{D}_{\mathscr{E}} \otimes \mathscr{L}$, and has isolated zeroes. If $\gamma \in \widehat{\mathfrak{Q}}_{\mathscr{E}}^{\bullet}$ is such that $\hat{\delta}_{V} \gamma=0$, we have

$$
\int_{X} \gamma(t)=\left(\frac{2 \pi i}{t}\right)^{n} \operatorname{Res}_{V, Z}\left(\hat{p}(\gamma)_{0}(t)\right)
$$

Proof. The proof of Theorem 3.2 works also in this case with only minor modifications. By using the identity (17) we reduce again the proof to the case $\mathscr{E}=\{0\}$. Note that in this case, $\widehat{Q}_{0}^{k}=\oplus_{p+q=k} \mathscr{L}^{-p+n} \otimes_{\mathscr{O}_{X}} \Omega_{X}^{p, q}$, so that $\widehat{Q}_{0}^{2 n}=\Omega_{X, \mathbb{C}}^{2 n}, \widehat{Q}_{0}^{0}=\mathscr{L}^{n}$.

We need to fix hermitian metrics both in $X$ and on $\mathscr{L}$, and define $\theta$ as the image of the complex conjugate of $V$ in $\Gamma\left(\Omega_{X}^{1,0} \otimes \mathscr{L}\right)$ via the induced hermitian metric on $\Theta_{X} \otimes \mathscr{L}$. The computation in the proof of Theorem 3.2 also works in this case; indeed, since the zeroes $x_{j}$ of $V$ are isolated, around any $x_{j}$ we can find a neighbourhood over which $\mathscr{L}$ trivializes.

Remark 4.2. Proposition 2.1 and Remark 3.4 can be extended to the meromorphic case, obtaining the cohomology complex and the localization formula (Theorem 6.6) described in Section 6 of [16].

4.3. Twisted moment maps. In the presence of the line bundle $\mathscr{L}$ we need to slightly modify the moment map construction of the previous section. As before, we fix an hermitian metric on $\mathscr{E}$, and denote by $K$ the curvature of the corresponding Chern connection. The moment map $\mu$ is again defined by equation (12), with $V$ a section of $\Theta_{X} \otimes \mathscr{L}$, and $\mathscr{E} \rightarrow \mathscr{E} \otimes \mathscr{L}$ is a differential operator which lifts it. This moment map enjoys the properties

(i) $\bar{\partial}_{H o m(\mathscr{E}, \mathscr{E} \otimes \mathscr{L})} \mu=\imath_{V} K$;

(ii) $\mu\left(x_{j}\right)=\mathbb{L}_{\tilde{V}, j}$ for all zeroes $x_{j}$ of $V$,

with $\mathbb{L}_{\tilde{V}, j}$ defined as in Lemma 3.1 . 
4.4. Carrell-Liebermann's formula. The Carrell-Liebermann formula is obtained from the formula (18) just making a specific choice of the cocycle $\gamma$. We assume that $\mathscr{L} \simeq \mathscr{O}_{X}(D)$ for an effective divisor $D$ in $X$. Thus we have maps $\mathscr{L}^{m_{1}} \rightarrow \mathscr{L}^{m_{2}}$ whenever $m_{1} \leq m_{2}$. This allows us to regard the traces of the powers of the equivariant curvature $\tilde{K}=K+t \mu$ as cocycles in $\widehat{\mathfrak{Q}}_{\mathscr{E}}^{\bullet}$.

Let $P$ be an Ad-invariant polynomial on the algebra $\operatorname{gl}(r, \mathbb{C})$ of $r \times r$ complex matrices, and let $\Phi$ be the polynomial expressing $P(a(\mathscr{E}))$ in terms of the Chern classes $c_{1}(\mathscr{E}), \ldots, c_{r}(\mathscr{E})$, i.e.,

$$
P(a(\mathscr{E}))=\Phi\left(c_{1}(\mathscr{E}), \ldots, c_{r}(\mathscr{E})\right) .
$$

After setting $\tilde{K}=K+t \mu$, we define the equivariant Chern forms of $\mathscr{E}$ as

$$
\tilde{c}_{k}(\mathscr{E})=P_{k}\left(\frac{i}{2 \pi} \tilde{K}\right)
$$

Then $\Phi\left(\tilde{c}_{1}(\mathscr{E}), \ldots, \tilde{c}_{r}(\mathscr{E})\right)$ is a cocycle in $\widehat{\mathfrak{Q}}_{\mathscr{E}}^{\bullet}$, and

$$
\int_{X} P(a(\mathscr{E}))=\int_{X} \Phi\left(\tilde{c}_{1}(\mathscr{E}), \ldots, \tilde{c}_{r}(\mathscr{E})\right)
$$

Finally, we note that evaluating the polynomial $P$ on the homomorphism $\mathbb{L}_{\tilde{V}, j}: \mathscr{E}_{x_{j}} \rightarrow$ $(\mathscr{E} \otimes \mathscr{L})_{x_{j}}$ we obtain an element $P\left(\mathbb{L}_{\tilde{V}, j}\right) \in \mathscr{L}_{x_{j}}^{n}$, and summing over all zeroes of $V$, we obtain a section $P\left(\mathbb{L}_{\tilde{V}}\right) \in H^{0}\left(Z, \mathscr{L}^{n}\right)$.

The localization formula (18) now gives:

Corollary 4.3 (Carrell-Liebermann's localization formula). Under the same hypotheses of Theorem 4.1, we have

$$
\int_{X} P(a(\mathscr{E}))=(2 \pi i)^{n} \operatorname{Res}_{V, Z}\left(P\left(\mathbb{L}_{\tilde{V}}\right)\right)
$$

Proof. We only need to check that if we make the choice $\gamma=\Phi\left(\tilde{c}_{1}(\mathscr{E}), \ldots, \tilde{c}_{r}(\mathscr{E})\right)$, then the residue of $p(\gamma)_{0}$ at $x_{j}$ coincides with $\operatorname{Res}_{V, Z_{j}}\left(P\left(\mathbb{L}_{\tilde{V}}\right)\right)$. This follows from the equality

$$
p\left(\tilde{c}_{k}(\mathscr{E})\right)_{0}=P_{k}\left(\frac{i}{2 \pi} \mu\right) .
$$

Remark 4.4. We can use Example 3.5 to show that our localization theorem is indeed more general than the Carrell-Liebermann formula. Indeed, if we take $\mathscr{E}=\mathscr{O}_{X}$, since the Atiyah class of $\mathscr{O}_{X}$ is zero, for every nonzero polynomial $P$ the Carrell-Liebermann formula (20) simply yields the identity (15), i.e.,

$$
\sum_{\ell} \frac{1}{J_{\ell}}=0
$$


(cf. Example 3.8). Note that is implied by equation (11) taking $\omega_{2}=0, f=1$.

4.5. Baum-Bott formula. Given a line bundle $\mathscr{L}$ on $X$, which we assume to be effective, Baum-Bott's meromorphic vector field theorem is a localization formula which expresses the Chern numbers of the virtual bundle $\Theta_{X}-\mathscr{L}^{*}$ as a sum of residues. One can deduce that formula from our general localization formula (18).

Let $V$ be a global holomorphic section of $\Theta_{X} \otimes \mathscr{L}$, that is, a meromorphic vector field on $X$, having isolated zeroes. In general, it does not lift to a differential operator $\tilde{V}: \Theta_{X} \rightarrow$ $\Theta_{X} \otimes \mathscr{L}$. One can try to define a lift locally, using a trivialization of $\mathscr{L}$ and extending the Lie derivative by linearity with respect to the coefficients in $\mathscr{L}$. The local expressions in general do not match, but the mismatch is a multiple of $V$, so that one obtains a welldefined object in $\operatorname{Hom}\left(\Theta_{X \mid Z},\left(\Theta_{X} \otimes \mathscr{L}\right)_{\mid Z}\right)$, where $Z$ is the zero-cycle of the zeroes of $V$, as before. If we evaluate a polynomial $P$ on this object, we obtain a well-defined residue. From one viewpoint, the content of Baum-Bott's meromorphic vector field theorem is that this residue computes a Chern number of the virtual bundle $\Theta_{X}-\mathscr{L}^{*}$.

We shall now offer a proof of this fact, basically following [7]. Let $\left\{U_{i}\right\}$ be an open cover over which $\mathscr{L}$ trivializes, and for each $i$, let $t_{i}$ be a generator of $\mathscr{L}\left(U_{i}\right)$. Moreover, let $\left\{\rho_{i}\right\}$ be a (smooth) partition of unity subordinated to $\left\{U_{i}\right\}$, and let $\omega_{i}$ be connection forms, each defined on $U_{i}$, of the Chern connection $\nabla$ for $\Theta_{X}$ given by an hermitian metric on $X$. We define

$$
\omega=\sum_{i} \rho_{i}\left(\omega_{i}-t_{i}^{-1} d t_{i} \otimes \operatorname{Id}_{\Theta_{X}}\right), \quad K=\bar{\partial} \omega, \quad \mu=\sum_{i} \rho_{i}\left(\tilde{V}_{i}-\nabla_{V}\right)
$$

where $\tilde{V}_{i}$ is the local lift of $V$ defined above. We have $K \in \Omega^{1,1}\left(\operatorname{End}\left(\Theta_{X}\right)\right), \mu \in \Gamma\left(\operatorname{End}\left(\Theta_{X}\right) \otimes\right.$ $\mathscr{L})$, and, as in Section $4.3, \bar{\partial} \mu=\imath_{V} K$. Note that if $\mathscr{L} \simeq \mathscr{O}_{X}$, one can make choices such that $K$ is the curvature of the Chern connection, and $\mu$ is a moment map. Now, a direct calculation shows the following.

Lemma 4.5. For every $k \geq 1$, the $(k, k)$-form $\operatorname{tr}\left(\left(\frac{i}{2 \pi} K\right)^{k}\right)$ is closed, and its cohomology class equals the $k$-th Chern character of the virtual bundle $\Theta_{X}-\mathscr{L}^{*}$.

Proposition 4.6. (Baum-Bott's formula) Let $V$ be a global section of $\Theta_{X} \otimes \mathscr{L}$ having isolated zeroes, and let $\Phi$ be a polynomial in $n$ variables whose degree in the $i$-th variable is $m_{i}$, and such that $\sum_{i=1}^{n} i m_{i}=n$. Denote by $\gamma_{i}, i=1, \ldots, n$, the Chern classes of the virtual bundle $\Theta_{X}-\mathscr{L}^{*}$, and by $\alpha_{1}^{(j)}, \ldots, \alpha_{n}^{(j)}$ the Chern classes of the linear morphisms 
$\left(\Theta_{X}\right)_{x_{j}} \rightarrow\left(\Theta_{X} \otimes \mathscr{L}\right)_{x_{j}}$ given by the Jacobian of $V$ at its zeroes. Then,

$$
\int_{X} \Phi\left(\gamma_{1}, \ldots, \gamma_{n}\right)=(2 \pi i)^{n} \sum_{j} \operatorname{Res}_{V, x_{j}}\left(\Phi\left(\alpha_{1}^{(j)}, \ldots, \alpha_{n}^{(j)}\right)\right) .
$$

Proof. As in Section 3.4, but using the endomorphism-valued forms $K$ and $\mu$ we have just defined, and taking Lemma 4.5 into account.

\section{REFERENCES}

[1] M. F. Aтіуaн, Complex analytic connections in fibre bundles, Trans. Amer. Math. Soc., 85 (1957), pp. 181-207.

[2] M. F. Atiyah And R. Bott, The moment map and equivariant cohomology, Topology, 23 (1984), pp. 1-28.

[3] P. Baum And R. Bott, On the zeroes of meromorphic vector fields, in Essays on Topology and Related Topics (Mémoires dédiés à Georges de Rham), Springer, New York, 1970, pp. 29-47.

[4] N. Berline, E. Getzler, And M. Vergne, Heat kernels and Dirac operators, vol. 298 of Grundlehren der Mathematischen Wissenschaften, Springer-Verlag, Berlin, 1992.

[5] R. Bотт, Vector fields and characteristic numbers, Michigan Math. J., 14 (1967), pp. 231-244.

[6] U. Bruzzo, L. Cirio, P. Rossi, And V. N. Rubtsov, Equivariant cohomology and localization for Lie algebroids, Funct. Anal. Appl., 43 (2009), pp. 18-29.

[7] J. B. Carrell, A remark on the Grothendieck residue map, Proc. Amer. Math. Soc., 70 (1978), pp. $43-48$.

[8] — Vector fields, residues and cohomology, in Parameter spaces (Warsaw, 1994), vol. 36 of Banach Center Publ., Polish Acad. Sci., Warsaw, 1996, pp. 51-59.

[9] J. B. Carrell And D. I. Lieberman, Vector fields and Chern numbers, Math. Ann, 225 (1977), pp. 263-273.

[10] S. Evens, J.-H. Lu, And A. Weinstein, Transverse measures, the modular class and a cohomology pairing for Lie algebroids, Quart. J. Math. Oxford, 50 (1999), pp. 417-436.

[11] H. FEnG AND X. MA, Transversal holomorphic sections and localization of analytic torsions, Pacific J. Math., 219 (2005), pp. 255-270.

[12] P. Griffiths And J. Harris, Principles of algebraic geometry, Wiley Classics Library, John Wiley \& Sons Inc., New York, 1994. Reprint of the 1978 original.

[13] R. Hartshorne, Residues and duality, Lecture Notes in Mathematics, No. 20, Springer-Verlag, Berlin, 1966.

[14] J. Huebschmann, Duality for Lie-Rinehart algebras and the modular class, J. Reine Angew. Math., 510 (1999), pp. 103-159.

[15] Y. LI, The equivariant cohomology theory of twisted generalized complex manifolds, Comm. Math. Phys., 281 (2008), pp. 469-497.

[16] K. LIU, Holomorphic equivariant cohomology, Math. Ann., 303 (1995), pp. 125-148. 